\title{
Is the concept of avian pathogenic Escherichia coli as a single pathotype fundamentally flawed?
}

\section{Charlotte Collingwood ${ }^{1}$, Kirsty Kemmett ${ }^{1}$, Nicola Williams ${ }^{2}$ and Paul Wigley ${ }^{1}$ *}

1 Department of Infection Biology, Institute of Infection and Global Health, School of Veterinary Science, University of Liverpool, Neston, UK

2 Department Epidemiology and Population Health, Institute of Infection and Global Health, School of Veterinary Science, University of Liverpool, Neston, UK

\section{Edited by:}

Guillermo Tellez, University of

Arkansas, USA

Reviewed by:

Raul G. Barletta, University of

Nebraska Lincoln, USA

Pierre Germon, National Institute for

Agricultural Research, France

${ }^{*}$ Correspondence:

Paul Wigley, University of Liverpool, Leahurst Campus, CH64 7TE Neston, UK

e-mail: paul.wigley@liv.ac.uk
Avian Pathogenic Escherichia coli (APEC) is a major pathogen within the poultry industry. However disease, especially in broiler chickens, may be caused by range of $E$. coli genotypes that carry few, if any, virulence factors associated with APEC. Furthermore, commensal $E$. coli in the intestines of healthy birds may carry an array of APEC virulence factors suggesting they have potential to cause disease when opportunity arises. Given the diseases caused by APEC, namely colibacillosis and salpingitis peritonitis syndrome, are syndromic in nature and the great diversity of the strains causing disease we suggest it is wrong to consider disease as the result of a single APEC pathotype. Whilst it is clear certain pathogenic E. coli can be considered as APEC, much of the disease-associated with $E$. coli in domestic poultry is as much a consequence of increased host susceptibility due to stress, immunosuppression, co-infection, or poor welfare. This leads to more "opportunistic" infections rather than the result of infection with a specific pathotype. As such the current use of the term APEC for all cases of E. coli infection in the chicken is fundamentally flawed.

Keywords: chicken, Escherichia coli, APEC, broiler chicken, eggs
Avian Pathogenic Escherichia coli (APEC) is both a primary and secondary pathogen of the chicken and other avian species (1). It is considered to be a member of the extra-intestinal pathogenic E. coli (ExPEC) along with human Uropathogenic (UPEC) and neonatal meningitis-associated E. coli (NMEC) that cause disease outside the intestine. APEC infection may occur in broiler (meat) chicken, turkey, and egg-laying sectors. In all sectors, infection is syndromic in nature. In the broiler chicken, APEC infections are considered to typically lead to colibacillosis; a syndrome that includes respiratory tract infection, air sacculitis, pericarditis, perihepatitis, splenomegaly, and swollen head syndrome. In mature laying hens, reproductive tract infection leading to salpingitis or salpingo-peritonitis syndrome (SPS) is common.

Avian pathogenic Escherichia coli is amongst the greatest health threats to the developed poultry industries and its emergence perhaps reflects the decrease in the prophylactic use of antimicrobials or their use as growth promoting agents. Furthermore, the close genetic relationship between APEC and other ExPEC associated with human disease along with evidence from experimental animal models have lead to suggestions that APEC may represent a zoonotic risk (2-8).

Whilst in recent years, APEC has become accepted as a primary pathogen rather than a consequence of respiratory or immunosuppressive viral infections, our understanding of APEC and its pathogenesis has remained relatively limited, due, at least in part, to its great diversity and genomic plasticity $(3,4,9-13)$. This variation is true of other ExPECs too (14). E. coli associated with human intestinal disease harbor certain defining virulence factors such as Type III secretion systems, Shiga-like toxins, and enterotoxins. ExPEC are, in general, less well defined in terms of their virulence determinants and are more variable between isolates. These virulence determinants include bacterial capsule that helps avoid host innate immunity, adhesins such as fimbriae involved in attachment to host cells and tissues, hemolysins that lyse host blood cells and multiple systems for acquisition of iron needed by the bacterium. In APEC, no single common virulence factor has been identified in all strains. Although certain genes associated with pathogenicity are common in APEC, including: iss associated with serum resistance (15), ibeA associated with invasion (16), and sitA associated with iron acquisition, they are not found in all isolates. Virulence genes including iss, iroN, and the iuc and cva operons are often associated with large plasmids including the Colicin V (ColV) plasmid (11). Certain E. coli serotypes such as $\mathrm{O} 1$ and $\mathrm{O} 78$ are more frequently associated with colibacillosis. Potentially these lineages harbor the genetic backbone required to acquire virulence mechanisms. Representatives of these serotypes have been genome sequenced and characterized $(17,18)$ and whilst there is little doubt these represent highly pathogenic variants of APEC, the reality is that in commercial broiler chickens colibacillosis is caused by a wide range of E. coli serotypes. Recently in our laboratory we have, for the first time, compared E. coli isolates causing colibacillosis within a broiler flock with those carried as intestinal commensals (19). These data showed that many colibacillosis - associated isolates carry few, if any, of the genes most commonly ascribed as APEC virulence factors. In essence, they have the genotype of "intestinal commensals." Equally, virulence genes may be found in "commensal" E. coli residing in the intestines of presumed healthy birds. Furthermore, isolates from cases of colibacillosis are not associated with any specific phylogenetic group. It appears that soon after 
hatch chicks acquire a diverse range of $E$. coli as part of their microbiota, likely to be sourced from the hatchery environment. Within this population are isolates carrying virulence-associated genes (1), though the frequency of such genes decreases as birds age (19). E. coli found in the intestinal tract are likely to form a reservoir of potential infection, we have previously termed such isolates as potential APEC or pAPEC (19), and may be associated with early infection and mortality. Such isolates could be considered opportunists in a compromised avian host as a consequence of production-related stress, immunosuppression, or prior infection $(1,19,20)$. Whilst opportunistic infections are likely to reduce in likelihood in older birds, they represent a clear risk in broiler production.

In laying hens, the situation is a little clearer. We have previously shown that APEC isolates from an outbreak of SPS in a commercial layer flock was caused by a single isolate that displayed features of both APEC and UPEC (2). Full genomic analysis of this isolate is ongoing. Recently, we have looked at the distribution of key virulence in genes in 188 SPS isolates from the UK through PCR. These findings suggest the majority possess iss, hlyF (a hemolysin), and the iron acquisition genes iucC and iroN but other virulence genes are less frequently found (Figure 1). Whilst within the 188 isolates there is variation, there does at least seem to be at least greater commonality of virulence factors than in colibacillosis, although seven of these isolates do not posses any of the genes screened for. This closer relationship between certain genotypes and disease and layers has also been described in Denmark where it appears certain lineages are common throughout the country, though there is still considerable diversity (21). Intriguingly in our UK-based studies although genes of the iuc aerobactin operon are found commonly (over 70\%) in both SPS isolates and in systemically isolated E. coli in colibacillosis, iss which was found in $83 \%$ SPS isolates was only found in $25 \%$ of broiler colibacillosis isolates whilst ibe, was found in more than $60 \%$ of broiler isolates but was found in $<25 \%$ of layer SPS isolates. This may be coincidence, but does suggest that different virulence factors may play roles causing what are very different diseases. The use of the subcutaneous infection model for cellulitis in day old chicks has recently identified pic, a serine protease, as a putative a virulence factor (22). However as yet, this is only an association with reduced virulence, has no mechanism ascribed and has not been identified in other models. A problem is that phenotypes of virulence factors in the chicken are poorly understood, partly as a consequence of the difficulty in reproducing experimental infections, in particular of the reproductive tract, so making our understanding of the mechanisms of APEC disease rather rudimentary. Recently developed infection models for SPS may clarify some of the virulence factors and mechanisms that underlie this disease syndrome $(23,24)$. However, such models rely on direct delivery to the reproductive tract and, in common with colibacillosis models, where delivery is direct to the air sacs (18, 25), may fail to detect important factors involved in the initiation of infection and colonization of tissues.

So one may pose the question should APEC be defined as any $E$. coli isolated from a diseased or sick bird or should the term be more narrowly defined? It is clear that certain well-characterized isolates can be defined as APEC. They can cause disease in experimental models and possess a range of virulence factors. However disease, and in particular colibacillosis, may result from infection with an isolate which bears few or none of the hallmarks by which we would define APEC, other than the fact it has caused disease. The

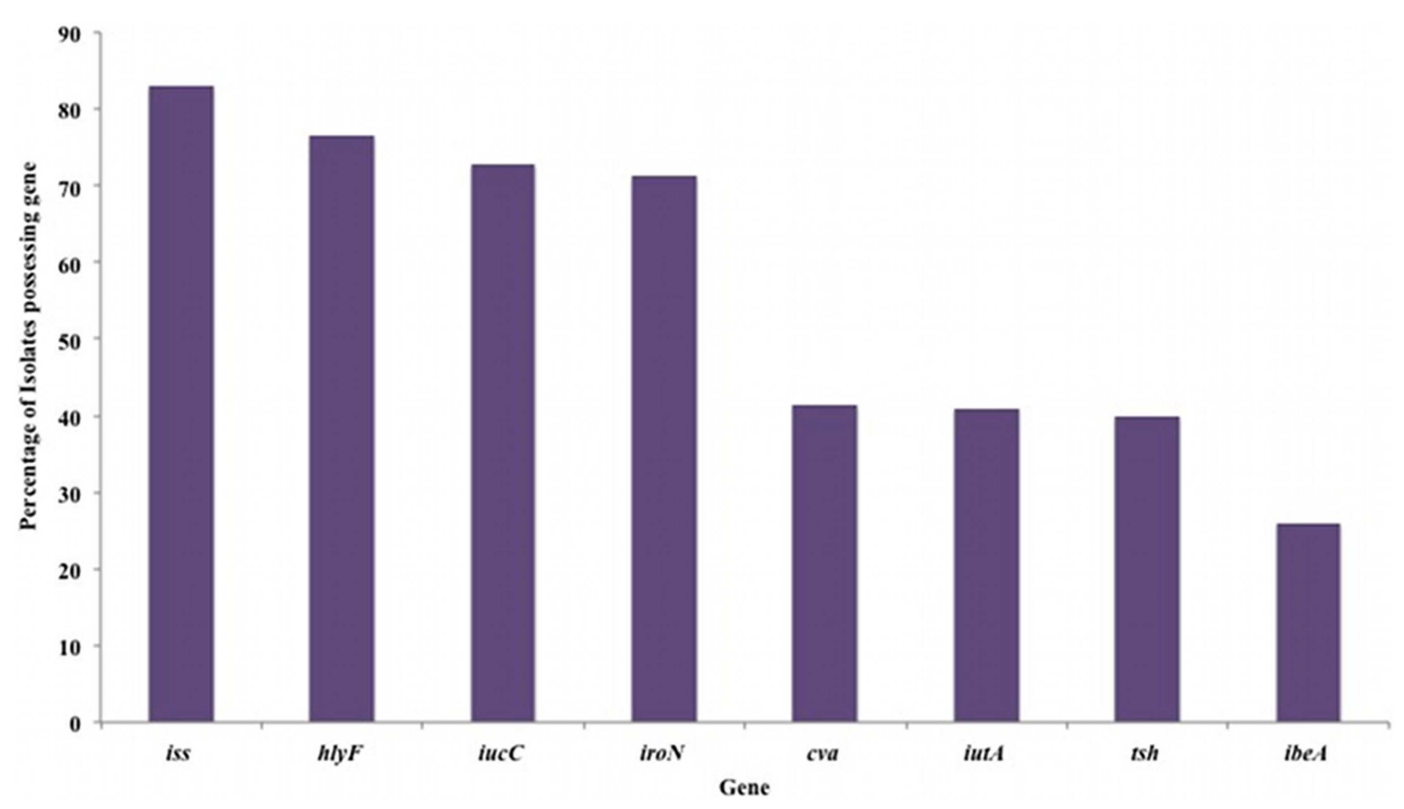

FIGURE 1 | Distribution of APEC associated virulence determinants in $188 \mathrm{E}$. coli isolates from cases of salpingitis peritonitis syndrome in UK laying hens. Virulence determinants detected by PCR using previously described methods $(13,19)$. The genes tested and their function were iss (increased serum survival), hlyF (a hemolysin), iucC (aerobactin), iroN (iron acquisition), cva (colicin operon), iutA (iron transport), tsh (a hemagglutinin), and ibeA (invasion). Data previously presented at American Society for Microbiology, Annual General Meeting, Denver, June 2013. 
use of the term APEC for E. coli that cause what in all likelihood is opportunistic infection in the chicken is fundamentally flawed. It is perhaps more appropriate to consider both colibacillosis and SPS as disease syndromes caused by an array of variable genotypes, that are as dependent on host susceptibility as any virulence factor possessed by the pathogen. It is probably fair to say APEC is successful pathogens that are far more likely to lead to disease in poultry than commensal E. coli. However, disease is not just restricted to those isolates we can define as having an APEC pathotype, and trying to pin all incidences of colibacillosis or SPS on an APEC pathotype is flawed. Of course such concepts are more challenging both to scientists and producers when identifying specific causative agents and their mechanisms are the norm.

There are also implications in disease control. It is difficult to control bacteria that are normally a commensal and although vaccination against APEC (26), especially in laying hens, has value it is not feasible to achieve either economically or immunologically in young broiler chicks. As such, good hatchery hygiene and management are important in controlling early mortalities, with good management and welfare likely to reduce the risk of colibacillosis in growing broilers. This also includes effective control of other pathogens including respiratory viruses where E. coli is a common secondary pathogen. Vaccination may not be effective against such a diverse microbial population and removal of $E$. coli as part of the microbiota may have other implications we cannot foresee. That said, approaches to sequence multiple genomes of E. coli from the chicken could reveal a common "core" genome in disease-associated E. coli, identifying a genetic relationship that cannot be found when considering virulence factors alone. Such an approach may also lead to the identification of novel targets for future vaccines.

In conclusion, we believe that E. coli disease in the chicken cannot be simply defined as being caused by a single pathotype of $E$. coli. In particular, colibacillosis is perhaps better defined as disease caused by E. coli rather than by Avian Pathogenic E. coli, and that the term APEC be reserved for the smaller number of well-defined "bona fide" pathogenic isolates with a range of defined virulence determinants that can reproduce disease in animal models. There are APEC, but not all disease-associated with $E$. coli in the chicken is caused by APEC.

\section{ACKNOWLEDGMENTS}

We wish to thank Dr. Tim Wallis and Ridgeway Biologicals for the panel of SPS-associated isolates. Charlotte Collingwood is supported by a BEMB Educational Trust Ph.D. award. Kirsty Kemmett was supported by a BBSRC DTG Ph.D. award.

\section{REFERENCES}

1. Antao EM, Glodde S, Li G, Sharifi R, Homeier T, Laturnus C, et al. The chicken as a natural model for extraintestinal infections caused by avian pathogenic Escherichia coli (APEC). Microb Pathog (2008) 45:361-9. doi:10.1016/j.micpath. 2008.08.005

2. Rodriguez-Siek KE, Giddings CW, Doetkott C, Johnson TJ, Fakhr MK, Nolan LK. Comparison of Escherichia coli isolates implicated in human urinary tract infection and avian colibacillosis. Microbiology (2005) 151:2097-110. doi:10.1099/mic. 0.27499-0

3. Moulin-Schouleur M, Schouler C, Tailliez P, Kao MR, Bree A, Germon P, et al. Common virulence factors and genetic relationships between O18:K1:H7
Escherichia coli isolates of human and avian origin. J Clin Microbiol (2006) 44:3484-92. doi:10.1128/JCM.00548-06

4. Moulin-Schouleur M, Reperant M, Laurent S, Bree A, Mignon-Grasteau S, Germon P, et al. Extraintestinal pathogenic Escherichia coli strains of avian and human origin: link between phylogenetic relationships and common virulence patterns. J Clin Microbiol (2007) 45:3366-76. doi:10.1128/JCM.00037-07

5. Johnson TJ, Wannemuehler Y, Johnson SJ, Stell AL, Doetkott C, Johnson JR, et al. Comparison of extraintestinal pathogenic Escherichia coli strains from human and avian sources reveals a mixed subset representing potential zoonotic pathogens. Appl Environ Microbiol (2008) 74:7043-50. doi:10.1128/ AEM.01395-08

6. Tivendale KA, Logue CM, Kariyawasam S, Jordan D, Hussein A, Li GW, et al. Avian-pathogenic Escherichia coli strains are similar to neonatal meningitis $E$. coli strains and are able to cause meningitis in the rat model of human disease. Infect Immun (2010) 78:3412-9. doi:10.1128/IAI.00347-10

7. Vincent C, Boerlin P, Daignault D, Dozois CM, Dutil L, Galanakis C, et al. Food reservoir for Escherichia coli causing urinary tract infections. Emerg Infect Dis (2010) 16:88-95. doi:10.3201/eid1601.091118

8. Mora A, Viso S, Lopez C, Alonso MP, Garcia-Garrote F, Dabhi G, et al. Poultry as reservoir for extraintestinal pathogenic Escherichia coli O45:K1:H7-B2ST95 in humans. Vet Microbiol (2013) 167:506-12. doi:10.1016/j.vetmic.2013. 08.007

9. La Ragione RM, Woodward MJ. Virulence factors of Escherichia coli serotypes associated with avian colisepticaemia. Res Vet Sci (2002) 73:27-35. doi:10.1016/ S0034-5288(02)00075-9

10. Ewers C, Janssen T, Kiessling S, Philipp HC, Wieler LH. Molecular epidemiology of avian pathogenic Escherichia coli (APEC) isolated from colisepticemia in poultry. Vet Microbiol (2004) 104:91-101. doi:10.1016/j.vetmic.2004. 09.008

11. Johnson TJ, Siek KE, Johnson SJ, Nolan LK. DNA sequence of a ColV plasmid and prevalence of selected plasmid-encoded virulence genes among avian Escherichia coli strains. J Bacteriol (2006) 188:745-58. doi:10.1128/JB.188.2.745-758.2006

12. Dziva F, Stevens MP. Colibacillosis in poultry: unravelling the molecular basis of virulence of avian pathogenic Escherichia coli in their natural hosts. Avian Pathol (2008) 37:355-66. doi:10.1080/03079450802216652

13. Timothy S, Shafi K, Leatherbarrow AH, Jordan FTW, Wigley P. Molecular epidemiology of a reproductive tract-associated colibacillosis outbreak in a layer breeder flock associated with atypical avian pathogenic Escherichia coli. Avian Pathol (2008) 37:375-8. doi:10.1080/03079450802216579

14. Manges AR, Tabor H, Tellis P, Vincent C, Tellier PP. Endemic and epidemic lineages of Escherichia coli that cause urinary tract infections. Emerg Infect Dis (2008) 14:1575-83. doi:10.3201/eid1410.080102

15. Foley SL, Horne SM, Giddings CW, Robinson M, Nolan LK. Iss from a virulent avian Escherichia coli. Avian Dis (2000) 44:185-91. doi:10.2307/1592523

16. Germon P, Chen YH, He L, Blanco JE, Bree A, Schouler C, et al. ibeA, a virulence factor of avian pathogenic Escherichia coli. Microbiology (2005) 151:1179-86. doi:10.1099/mic.0.27809-0

17. Johnson TJ, Kariyawasam S, Wannemuehler Y, Mangiamele P, Johnson SJ, Doetkott C, et al. The genome sequence of avian pathogenic Escherichia coli strain O1: K1: H7 shares strong similarities with human extraintestinal pathogenic E. coli genomes. J Bacteriol (2007) 189:3228-36. doi:10.1128/JB.00537-07

18. Dziva F, Hauser H, Connor TR, van Diemen PM, Prescott G, Langridge GC, et al. Sequencing and functional annotation of avian pathogenic Escherichia coli serogroup O78 strains reveal the evolution of E. coli lineages pathogenic for poultry via distinct mechanisms. Infect Immun (2013) 81:838-49. doi:10.1128/IAI.00585-12

19. Kemmett K, Humphrey T, Rushton S, Close A, Wigley P, Williams NJ. A longitudinal study simultaneously exploring the carriage of APEC virulence associated genes and the molecular epidemiology of faecal and systemic E. coli in commercial broiler chickens. PLoS ONE (2013) 8:e67749. doi:10.1371/journal. pone.0067749

20. Kemmett K, Williams NJ, Chaloner G, Humphrey S, Wigley P, Humphrey T. The contribution of systemic Escherichia coli infection to the early mortalities of commercial broiler chickens. Avian Pathol (2014) 43:37-42. doi:10.1080/ 03079457.2013 .866213

21. Pires-dos-Santos T, Bisgaard M, Christensen H. Genetic diversity and virulence profiles of Escherichia coli causing salpingitis and peritonitis in broiler breeders. Vet Microbiol (2013) 162:873-80. doi:10.1016/j.vetmic.2012.11.008 
22. Barbieri NL, de Oliveira AL, Tejkowski TM, Pavanelo DB, Rocha DA, Matter LB, et al. Genotypes and pathogenicity of cellulitis isolates reveal traits that modulate APEC virulence. PLOS ONE (2013) 8:e72322. doi:10.1371/ journal.pone.0072322

23. Chaudhari AA, Kariyawasam S. An experimental infection model for Escherichia coli egg peritonitis in layer chickens. Avian Dis (2014) 58:25-33. doi:10.1637/ 10536-032213-Reg.1

24. Pors SE, Olsen RH, Christensen JP. Variations in virulence of avian pathogenic Escherichia coli demonstrated by the use of a new in vivo infection model. Vet Microbiol (2014) 170:368-74. doi:10.1016/j.vetmic.2014.02.043

25. Sadeyen JR, Kaiser P, Stevens MP, Dziva F. Analysis of immune responses induced by avian pathogenic Escherichia coli infection in turkeys and their association with resistance to homologous re-challenge. Vet Res (2014) 45:19. doi:10.1186/1297-9716-45-19

26. La Ragione RM, Woodward MJ, Kumar M, Rodenberg J, Fan H, Wales AD, et al. Efficacy of a live attenuated Escherichia coli O78:K80 vaccine in chickens and turkeys. Avian Dis (2013) 57:273-9. doi:10.1637/10326-081512-Reg.1
Conflict of Interest Statement: The authors declare that the research was conducted in the absence of any commercial or financial relationships that could be construed as a potential conflict of interest.

Received: 30 May 2014; accepted: 28 June 2014; published online: 14 October 2014. Citation: Collingwood C, Kemmett K, Williams N and Wigley P (2014) Is the concept of avian pathogenic Escherichia coli as a single pathotype fundamentally flawed? Front. Vet. Sci. 1:5. doi: 10.3389/fvets.2014.00005

This article was submitted to Veterinary Infectious Diseases, a section of the journal Frontiers in Veterinary Science.

Copyright (C) 2014 Collingwood, Kemmett, Williams and Wigley. This is an open-access article distributed under the terms of the Creative Commons Attribution License (CC $B Y)$. The use, distribution or reproduction in other forums is permitted, provided the original author(s) or licensor are credited and that the original publication in this journal is cited, in accordance with accepted academic practice. No use, distribution or reproduction is permitted which does not comply with these terms. 